\title{
Whole Genome Amplification by T7-Based Linear Amplification of DNA (TLAD): III. Sample Purification
}

\author{
Chih Long Liu, Bradley E. Bernstein, and Stuart L. Schreiber
}

This protocol was adapted from "DNA Linear Amplification," Chapter 7, in Whole Genome Amplification: Methods Express (eds. Hughes and Laskin), from the Methods Express series. Scion Publishing Ltd., Oxfordshire, UK, 2005.

\section{INTRODUCTION}

T7-based linear amplification of DNA (TLAD) uses a linear amplification approach based on in vitro transcription (IVT) of template DNA by RNA polymerase from the T7 phage. TLAD was designed primarily for use with the ChIP-chip method (whereby DNA recovered from chromatin immunoprecipitation [ChIP] of cell lysate is used for subsequent analysis on DNA microarrays) and requires nanogram quantities of dsDNA to generate microgram amounts of amplified RNA. Briefly, the strategy is to add a $3^{\prime}$ conserved end to the template dsDNA, using terminal deoxynucleotidyl transferase (TdT) tailing, which permits the addition of a T7 promoter sequence in the subsequent second-strand synthesis step. IVT can then use this newly appended T7 promoter and linearly amplify the template dsDNA, producing antisense RNA (aRNA) product. After the IVT reaction is complete, the aRNA is cleaned up using the QIAGEN RNeasy Kit. This protocol for RNA sample purification is based on the manufacturer's protocol for cleaning up RNA reactions, with minor modifications.

\section{RELATED INFORMATION}

Information about ChIP-chip and an overview of the TLAD method (including suggested controls and interpretation of results) is provided in Whole Genome Amplification by T7-Based Linear Amplification of DNA (TLAD): Overview (Liu et al. 2008a). The articles Whole Genome Amplification by T7-Based Linear Amplification of DNA (TLAD): I. CIP Treatment of Samples and Tailing Reaction with Terminal Transferase (Liu et al. 2008b) and Whole Genome Amplification by T7-Based Linear Amplification of DNA (TLAD): II. Second-Strand Synthesis and In Vitro Transcription (Liu et al. 2008c) detail Parts I and II, respectively, of the TLAD method. TLAD was originally described by Liu et al. (2003); a schematic of the method is shown in Figure 1.

\section{MATERIALS}

CAUTIONS AND RECIPES: Please see Appendices for appropriate handling of materials marked with $\langle!>$, and recipes for reagents marked with $<\mathbf{R}>$.

\section{Reagents}

Agarose gel (1\%-2\%)

$<$ ! $>\beta$-Mercaptoethanol (14.2 M)

Ethanol (95\%-100\%) (RNase-free)

RNA sample from Whole Genome Amplification by T7-Based Linear Amplification of DNA

(TLAD): II. Second-Strand Synthesis and In Vitro Transcription (Liu et al. 2008c) 
RNeasy Mini kit (containing RNeasy columns; Buffer RLT; Buffer RPE; RNase-free $\mathrm{H}_{2} \mathrm{O}$; 2-mL RNase-free collection tubes; 1.5-mL RNase-free collection tubes) (QIAGEN)

Add $95 \%$ or $100 \%$ RNase-free ethanol to Buffer RPE before use; see manufacturer's protocol. If necessary, $80 \%$ ethanol may be substituted, although this has not been extensively tested. It may be necessary to order additional Buffer RPE separately, as this protocol consumes 50\% more Buffer RPE than the manufacturer's standard protocol.

\section{Equipment}

Equipment for running agarose gel

Microcentrifuge

Microcentrifuge tubes (1.5-mL RNase-free)

Pipette tips (low-retention, aerosol-barrier, RNase-free)

Spectrophotometer

Vortex mixer

\section{METHOD}

1. Prepare the Buffer RLT master mix by combining the following in a $1.5-\mathrm{mL}$ RNase-free microcentrifuge tube for each IVT reaction:

$350 \mu \mathrm{L} \quad$ Buffer RLT

$80 \mu \mathrm{L} \quad \mathrm{H}_{2} \mathrm{O}$

$3.5 \mu \mathrm{L} \quad \beta$-Mercaptoethanol

This master mix can be prepared up to a week in advance and aliquoted into 1.5- $\mathrm{mL}$ microcentrifuge tubes.

2. Transfer the contents of the IVT reaction from Whole Genome Amplification by T7-Based Linear Amplification of DNA (TLAD): II. Second-Strand Synthesis and In Vitro Transcription (Liu et al. 2008c) to the mix prepared in Step 1, and vortex briefly.

Low-retention, aerosol-barrier, RNase-free pipette tips are highly recommended here, because the RNA concentration in the IVT reaction tubes may be as high as $5 \mu \mathrm{g} / \mu \mathrm{L}$.

3. Add $250 \mu \mathrm{L}$ of ethanol (95\%-100\%) and mix well by pipetting. Do not centrifuge the tubes.

4. Apply the sample $(\sim 700 \mu \mathrm{L})$ to an RNeasy mini spin column sitting in a collection tube. Centrifuge for $15 \mathrm{sec}$ at $8000 \mathrm{~g}$. Discard flow-through.

5. Transfer the RNeasy column to a new 2-mL collection tube. Add $500 \mu \mathrm{L}$ of Buffer RPE (which must contain ethanol) and centrifuge for $15 \mathrm{sec}$ at $8000 \mathrm{~g}$. Discard flow-through but re-use the collection tube.

6. Pipette $500 \mu \mathrm{L}$ of Buffer RPE into the RNeasy column and centrifuge for $1 \mathrm{~min}$ at maximum speed in a microcentrifuge.

7. Remove flow-through and pipette another $500 \mu \mathrm{L}$ of Buffer RPE onto the column. Centrifuge for 2 min at maximum speed in a microcentrifuge to dry the column completely.

This is an additional wash that is not included in the QIAGEN protocol. If the RNA is to be used for microarray work or other applications involving fluorescence, this additional wash is necessary to remove remaining trace amounts of guanidine thiocyanate that would otherwise contaminate the eluted RNA and cause increased background noise in fluorescence applications such as microarrays.

8. Transfer the RNeasy column into a new 1.5-mL RNase-free collection tube, taking care not to carry over any flow-through from the 2-mL collection tube.

9. Add $30 \mu \mathrm{L}$ of $\mathrm{H}_{2} \mathrm{O}$ directly onto the membrane of the RNeasy column. Centrifuge for $1 \mathrm{~min}$ at $8000 \mathrm{~g}$ to elute. Repeat this step if the expected yield is $\geq 30 \mu \mathrm{g}$.

10. Check RNA concentration and quality by measuring the absorbance at $260 \mathrm{~nm}$ and $260 / 280 \mathrm{~nm}$, and by running a sample on a $1 \%-2 \%$ agarose gel. 


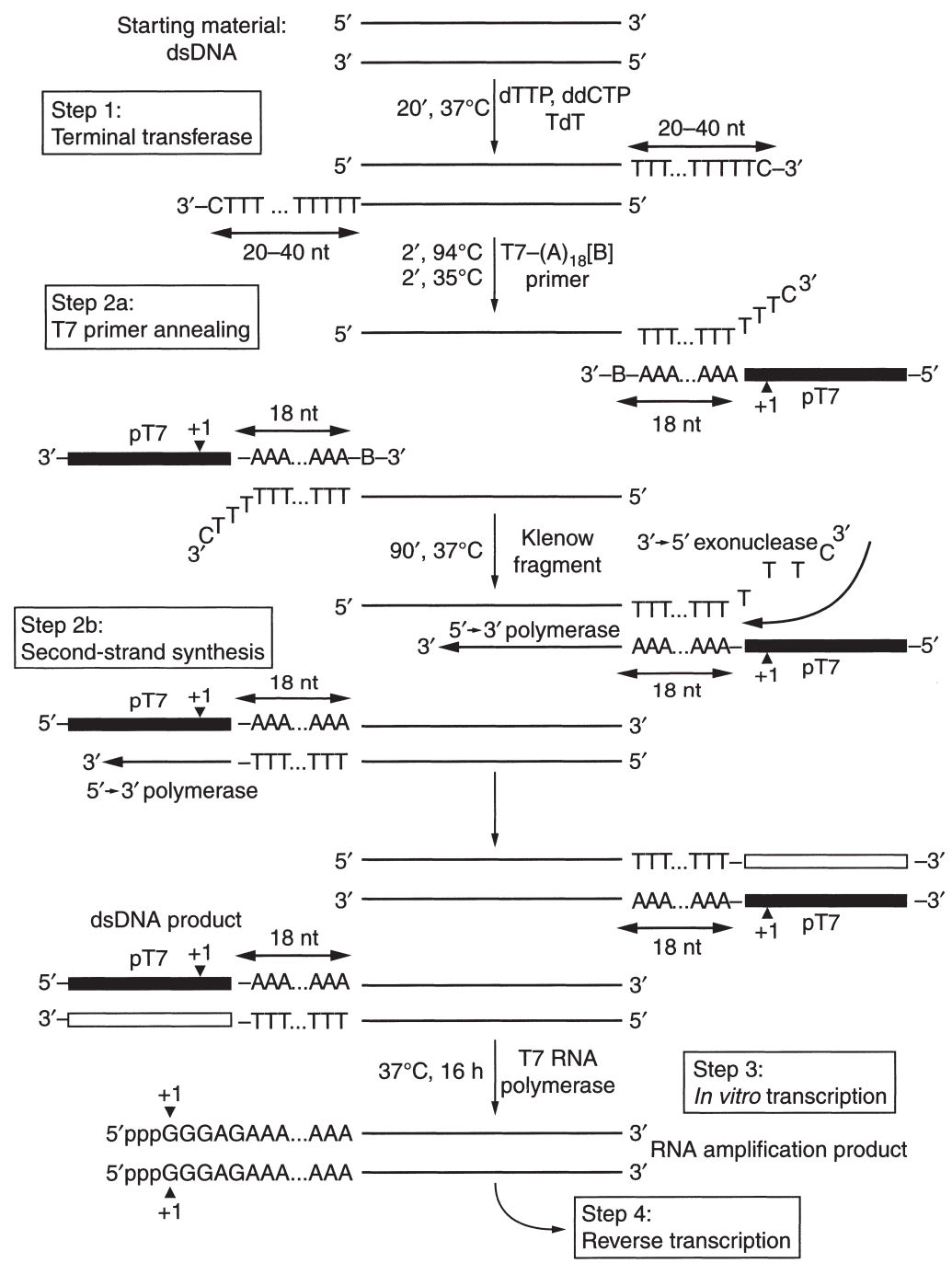

FIGURE 1. General strategy for the TLAD method. Starting with dsDNA template, TdT is used to add a poly(dT) tail to the 3 '-ends of the template. This tail subsequently provides a conserved binding site for the annealing of T7 promoter (pT7)-poly(dA) primer adapters. Following subsequent second-strand synthesis using the large fragment of DNA polymerase I (Klenow fragment), one pair of dsDNA templates, with each pair member representing one of the two complementary strands of the dsDNA, is generated, with a T7 promoter at the $5^{\prime}$-end of the amplicon. In the subsequent IVT step, RNA is transcribed from this template in an isothermal reaction, producing an RNA amplification product consisting of both strands of the original dsDNA template in high microgram quantities. Note that each RNA strand will contain a short sequence from the T7 promoter and a poly(A) tract, $5^{\prime}$ relative to the amplicon. (Reprinted with permission from Liu et al. [2003].)

A denaturing gel should only be used if the size distribution of the aRNA needs to be determined accurately. Figure 2 (lane 4) indicates a typical 2\% gel result. For information about interpreting results, see Whole Genome Amplification by T7-Based Linear Amplification of DNA (TLAD): Overview (Liu et al. 2008a).

See Troubleshooting.

If the purified aRNA product is to be used for subsequent microarray experiments, prime the reverse transcription reaction with random hexamer primers, for example, $5 \mu \mathrm{g} d(\mathrm{~N})$. The presence of oligo $(d T)$ primers will not interfere with reverse transcription, but oligo(dT) primers will not prime the reaction correctly.

\section{TROUBLESHOOTING}

The TLAD protocols in this set (Parts I, II, and III; see Related Information) routinely work well when the samples used are within the recommended starting amount range, and particularly when users 


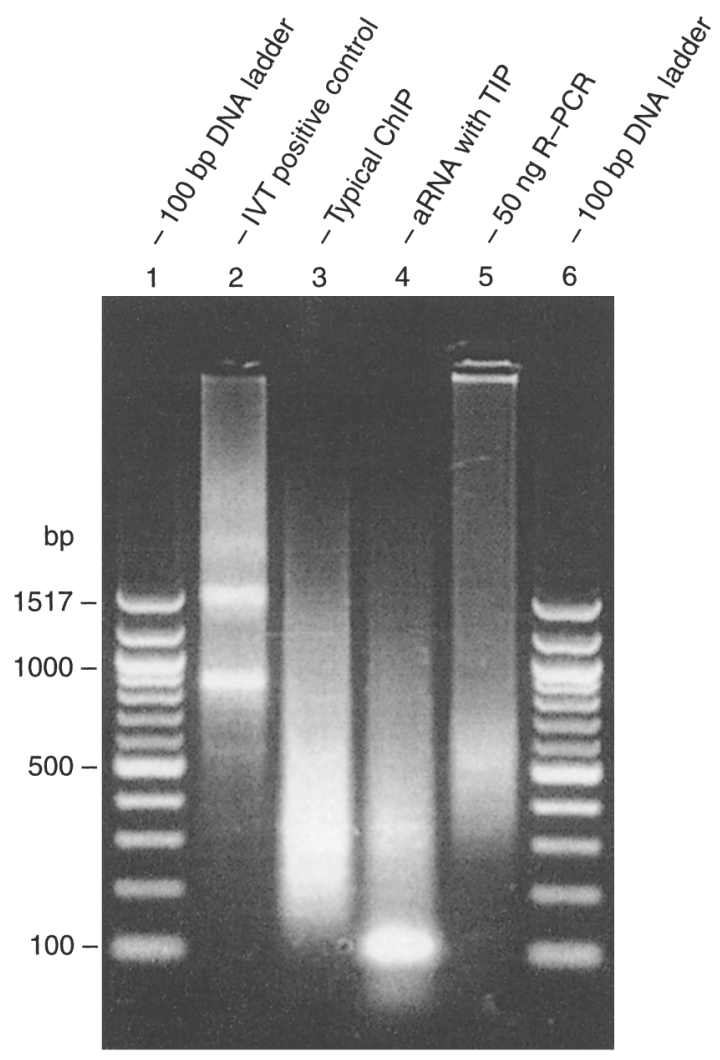

FIGURE 2. Amplification products on a nondenaturing $2 \%$ agarose gel in TAE buffer, stained with ethidium bromide. A 100-bp ladder (500 ng; New England Biolabs) or 650 ng of samples were loaded. (Lanes 1,6) 100-bp ladder; (lanes 24) aRNA amplified using the TLAD method, with the following templates: (lane 2) IVT positive control (pTRI-Xef); (lane 3) a typical ChIP sample (Saccharomyces cerevisiae bis-acetyl histone H3 on lysine 9 and 14) from sonicated genomic DNA; (lane 4) amplification from $5 \mathrm{ng}$ of a genomic digest of $S$. cerevisiae with Rsal, showing a strong band of templateindependent product (TIP) of $\sim 100 \mathrm{bp}$ (see Troubleshooting); (lane 5) the R-PCR product, for comparison, from a 50-ng amplification of the same template used to generate aRNA in lane 4 . The most remarkable attribute of lane 5 is the near absence of DNA smaller than 250 bp. (Reprinted with permission, (c) 2005 Scion Publishing Ltd.)

new to these protocols use the controls described in Whole Genome Amplification by T7-Based Linear Amplification of DNA (TLAD): Overview (Liu et al. 2008a). Poor aRNA yield is the most frequently encountered problem. Occasionally, one may encounter more subtle problems, either stemming from the nature and composition of the template DNA used or in ensuring compatibility with downstream applications such as microarrays.

Problem: The yield of aRNA is poor.

[Step 10]

Solution: Examination of the controls can quickly pinpoint the likely cause of the problem. Address the control results as follows:

\section{RNase Contamination}

The IVT control in Whole Genome Amplification by T7-Based Linear Amplification of DNA (TLAD): Overview (Liu et al. 2008a) provides a good way of determining whether there are any problems associated with handling RNA and maintaining an RNase-free environment. If the IVT control produces a poor yield, this may be due to contamination with RNases. This can be confirmed by running a $2 \%$ nondenaturing agarose gel in Tris-acetate-EDTA (TAE) and ethidium bromide. An RNase-contaminated IVT sample will yield a smear of low-molecular-weight material. If RNase contamination is determined to be the cause, ensure that aerosol-barrier, RNase-free pipette tips are used and that working surfaces are treated with RNaseZap (Ambion) or other RNase-decontaminating agents. This is particularly important if working with ChIP samples. To ensure that the RNase A used to digest RNA carried over 
from the ChIP is completely eliminated, use the pTRI-Xef linear plasmid supplied with the Ambion IVT kit, add RNase A to the amount typically used for post-ChIP RNA digestion, and then carry it through three successive cleanups using the MinElute Reaction Cleanup kit as in Steps 3-8 of Whole Genome Amplification by T7-Based Linear Amplification of DNA (TLAD): I. CIP Treatment of Samples and Tailing Reaction with Terminal Transferase (Liu et al. 2008b).

\section{Suboptimal IVT Conditions}

If no RNase contamination is detected, either via the $A_{260} / A_{280}$ ratio from a UV absorbance reading of the sample, or from analyzing the sample by gel electrophoresis, it is likely that there are problems with the IVT reaction conditions:

- The NTP mix may have gone through too many freeze-thaw cycles. NTPs are very sensitive to freeze-thaw cycles, and each one decreases the yield. If this is the case, use a fresh IVT kit and aliquot the NTP mix (into more than four aliquots if necessary) before use as described in Whole Genome Amplification by T7-Based Linear Amplification of DNA (TLAD): II. Second-Strand Synthesis and In Vitro Transcription (Liu et al. 2008c).

- Excessive evaporation of the reaction volume may have occurred during incubation. The IVT incubation conditions have been designed to limit evaporation and vapor volume during the long incubation period. Follow the conditions described in Whole Genome Amplification by T7Based Linear Amplification of DNA (TLAD): II. Second-Strand Synthesis and In Vitro Transcription (Liu et al. 2008c). Using mineral oil is not recommended because it may interfere with either the IVT reaction or the aRNA cleanup (Steps 1-10 above), or both.

- The troubleshooting section in the manufacturer's manual accompanying the IVT kit may also be consulted.

\section{Poor Yield with the Positive Amplification Control}

This is likely to occur if the considerations described in Whole Genome Amplification by T7-Based Linear Amplification of DNA (TLAD): Overview (Liu et al. 2008a) are not followed.

- If the template DNA has a large proportion of 3' recessed ends, this template will not tail efficiently. A fill-in reaction using Klenow enzyme is recommended. ChIP samples from sheared genomic DNA typically have approximately half of their ends 3' recessed; this correspondingly reduces the yield by half. Double the starting amount or use Klenow fill-in to obtain yields comparable with those indicated in Table 1 of Whole Genome Amplification by T7-Based Linear Amplification of DNA (TLAD): Overview (Liu et al. 2008a).

- If the template DNA has a large proportion of 3' phosphate groups, the template will not tail efficiently. Note that some DNA fragmentation methods (such as restriction digestion) may leave behind 3 ' phosphate groups. Because this information is not always readily available for a given restriction enzyme, treat the template DNA with an alkaline phosphatase, as described in Whole Genome Amplification by T7-Based Linear Amplification of DNA (TLAD): I. CIP Treatment of Samples and Tailing Reaction with Terminal Transferase (Liu et al. 2008b), and see whether this solves the yield problem.

- Check the recovery yield of the template DNA after purification(s) using MinElute columns. From $50 \mathrm{ng}$ of input DNA, a yield of $50 \%-80 \%$ is typical. If the yields obtained are significantly less than this, verify that the column membrane is completely wetted by Buffer EB during the elution step. Furthermore, verify that the columns are viable-from 2004, QIAGEN required that the MinElute columns be stored at $4^{\circ} \mathrm{C}$ when not in use.

\section{Formation of Template-Independent Product}

The amplification product may contain a substantial amount of template-independent product when the mass ratio of T7 primer to template DNA significantly exceeds 5:1. This can easily occur when the starting amount of the template DNA is significantly overestimated. An example of this template-independent product is shown in Figure 2 (lane 4). We speculate that excess T7 primer during secondstrand synthesis produces primer dimers, which yields an IVT template that produces the band of low-molecular-weight material shown in Figure 2. This IVT side reaction diminishes the yield of the true amplification product. To prevent this from happening, follow Table 1 in Whole Genome Amplification by T7-Based Linear Amplification of DNA (TLAD): II. Second-Strand Synthesis and In Vitro 
Transcription (Liu et al. 2008c), which indicates the proper volumes and concentrations of T7 primer and template DNA to use for this step. If necessary, follow the recommendations discussed in Whole Genome Amplification by T7-Based Linear Amplification of DNA (TLAD): Overview (Liu et al. 2008a) for accurate measurement of the starting amount of template DNA.

Problem: Poly(A) tracts appear in the amplified RNA.

[Step 10]

Solution: The TdT reaction produces poly(dT) tails within a size range of 20-40 nucleotides (nt). While we speculate that overhanging $3^{\prime}$ regions of the poly(dT) tails in the subsequent second-strand synthesis are removed via the $3^{\prime}$ exonuclease activity of the Klenow fragment enzyme, there exists the possibility that the T7 primer may anneal in such a way that the 3' anchor of the primer (denoted by base designation "[B]" in Fig. 1) may not be base-paired and that the rest of the poly(dA) region of the primer is base-paired anywhere along the length of the poly(dT) tail. The resulting size distribution may thus be larger than the original template, usually in the order of 20-40 bp, and would appear as a gel shift and a broadening of the gel bands corresponding to that size range. This issue has not been examined carefully because the potential variability in tail length does not appear to affect amplification efficiency and fidelity when used on microarrays. However, this issue may be important for consideration of applications that are sensitive to this potential variability in tail lengths and to the poly $(\mathrm{A})$ tracts that will appear in the final amplification product.

Problem: dsRNA formation is observed in the amplified RNA product.

[Step 10]

Solution: Probe self-hybridization is unlikely to be an issue unless the probe composition is of low complexity (see Discussion). If dsRNA does form in significant proportions, it may also reduce the efficiency and yield of reverse transcription, either by slowing down the reverse transcriptase enzyme in the reverse transcription step or by causing insufficient denaturing of the dsRNA, leading to less efficient primer annealing. To compensate, we suggest using 50\% more RNA than the amount typically used for microarray probe labeling. Nevertheless, we have not found it necessary to investigate carefully the potential impact of aRNA self-hybridization on reverse transcriptase efficiency, because high amplification fidelity is typically obtained. Furthermore, we have found in many cases that foregoing this increase will still yield a lower but usable net signal intensity for most spotted microarray hybridizations.

\section{DISCUSSION}

Theoretically, dsRNA can conceivably form from the amplification products, since aRNA based on both strands of the original template is produced. However, we have not tested whether dsRNA actually forms under the conditions outlined in this method. For spotted microarray experiments, if DNA probe produced from the aRNA product undergoes some degree of self-hybridization, the end result could potentially be a decrease in net signal intensity or compression of the dynamic range in the ratiometric data obtained, or both. We have observed this in one case with a yeast open reading frame microarray where, in a low-complexity mixture containing less than 300 unique DNA species, amplification of both strands compressed the dynamic range by $\sim 60 \%-70 \%$ when compared with that obtained with a single-strand amplification (R. Butcher, pers. comm.). We were able to make this determination via single-strand amplification because the starting material already had conserved sequences that were different on each end of the amplicon. The protocols in this set normally do not provide that opportunity.

We believe this issue, however, should not have a significant impact on most studies that amplify highly complex mixtures of DNA, such as randomly fragmented genomic DNA. The amplification fidelity and signal quality have already been demonstrated to be at least as good as direct, unamplified Klenow labeling and better than R-PCR (Liu et al. 2003). We speculate that in a microarray hybridization, a DNA probe synthesized from a high-complexity mixture of aRNA (such as from amplification of sheared genomic DNA) is less likely to be affected by probe self-hybridization than probe synthesized from a low-complexity mixture (such as from amplification of a transcription factor ChIP that localizes to a small number of locations within the genome). The reason is that, during the hybridization process, a given probe strand in a highly complex mixture is more likely to hybridize to its complementary target on the microarray than to its complementary probe strand floating free in 
solution. This is because the complementary target on the microarray is fixed in location, while the complementary probe strand is free-floating and migrating throughout the hybridization solution. Thus, we believe that only in the case where probe composition is of low complexity should the user be concerned about probe self-hybridization.

\section{ACKNOWLEDGMENTS}

C.L.L. is supported by a Graduate Research Fellowship from the National Science Foundation. S.L.S. is an investigator at the Howard Hughes Medical Institute. B.E.B. is supported by a K08 Development Award from the National Cancer Institute. This work was supported by a grant from the National Institute for General Medical Sciences.

\section{REFERENCES}

Liu, C.L., Schreiber, S.L., and Bernstein, B.E. 2003. Development and validation of a T7 based linear amplification for genomic DNA. BMC Genomics 4: 19 doi: 10.1186/1471-2164-4-19.

Liu, C.L., Bernstein, B.E., and Schreiber, S.L. 2008a. Whole genome amplification by T7-based linear amplification of DNA (TLAD): Overview. CSH Protocols (this issue) doi: 10.1101/pdb.top42.

Liu, C.L., Bernstein, B.E., and Schreiber, S.L. 2008b. Whole genome amplification by T7-based linear amplification of DNA (TLAD): I. CIP treatment of samples and tailing reaction with terminal transferase. CSH Protocols (this issue) doi: 10.1101/pdb.prot5002.

Liu, C.L., Bernstein, B.E., and Schreiber, S.L. 2008c. Whole genome amplification by T7-based linear amplification of DNA (TLAD): II. Second-strand synthesis and in vitro transcription. CSH Protocols (this issue) doi: 10.1101/pdb.prot5003. 
Whole Genome Amplification by T7-Based Linear Amplification of DNA (TLAD): III. Sample Purification

Chih Long Liu, Bradley E. Bernstein and Stuart L. Schreiber

Cold Spring Harb Protoc; doi: 10.1101/pdb.prot5004

\begin{tabular}{cc}
\hline $\begin{array}{r}\text { Email Alerting } \\
\text { Service }\end{array}$ & Receive free email alerts when new articles cite this article - click here. \\
\hline $\begin{array}{c}\text { Subject } \\
\text { Categories }\end{array}$ & Browse articles on similar topics from Cold Spring Harbor Protocols. \\
& Bioinformatics/Genomics, general (192 articles) \\
& DNA Sequencing (96 articles) \\
& Genetic Variation (86 articles) \\
& Genetics, general (374 articles) \\
& Genome Analysis (191 articles) \\
& Genomic DNA (135 articles) \\
& Genomic Libraries (66 articles) \\
& Immunoprecipitation (75 articles) \\
& Libraries (147 articles) \\
& Libraries, general (113 articles) \\
& Molecular Biology, general (1293 articles) \\
RNA (317 articles) & RNA, general (269 articles) \\
&
\end{tabular}

